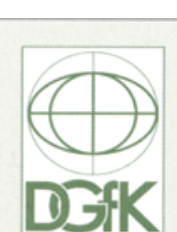

KN Kartographische Nachrichten Journal of Cartography and Geographic Information 66. Jahrgang, April 2016, Heft 3 Herausgeber: Deutsche Gesellschaft für Kartographie e.V. (DGfK) - Gesellschaft für Kartographie und Geomatik, vertreten durch den Präsidenten Prof. Dr. Manfred Weisensee

Organ der Deutschen Gesellschaft für Kartographie e.V. (DGfK), der Schweizerischen Gesellschaft für Kartografie (SGK) und der Österreichischen Kartographischen Kommission (OKK) in der Österreichischen Gesellschaft für Geographie (ÖGG). Schriftleitung

Hauptschriftleiter

Prof. Dr. rer. nat. habil. Mark Vetter (zuständig für Aufsätze, Berichte). Hochschule Karlsruhe - Technik und Wirtschaft, Moltkestr. 30, 76133 Karlsruhe; redaktion@kartographischenachrichten.de, Fon: 0049(0) 7219252599

Weitere Schriftleiter

Prof. Dr. phil. Frank Dickmann (Zweiter Schriftteiter, zustăndig für Nachrichten aus Hochschulen und Institutionen, Rezensionen).

Ruhr-Universităt Bochum, Geographisches Institut,

Postfach, 44780 Bochum; frank.dickmann@rub.de

Fon: 0049(0) 234 32-23379; Fax: 0049(0) 234 32-14964

Dipl.-Ing. Andreas Gollenstede (zustăndig für Geoinformation

aktuell) Haareneschstr. 91, 26121 Oldenburg; kn@gollenstede.com

Fon: 0049(0) 441 7779-545; Fax: 0049(0) 441 7779-750

Dr.-Ing. Thomas Chudy (zuståndig für DGfK-, SKG- und ÖGG-

Nachrichten). Martin-Luther-Universitāt Halle-Wittenberg, Institut

für Agrar- und Ernährungswissenschaften, Karl-Freiherr-von-

Fritsch-Straße 4, 06120 Halle/Saale, Fon: 0049(0) 3455522448 ;

Fax: 0049(0) 3455527109

Prof. Dr. Reinhard Zölitz (zuståndig für Nachrichten aus Hoch-

schulen und Institutionen), Institut für Geographie und Geologie

Kartographie und GIS, Friedrich-Ludwig-Jahn-Str. 16,

D-17489 Greifswald, Fon 03834864523 , Fax: 03834864501 ,

E-Mail:zoelitz@uni-greifswaldde

Mitarbeit für die Schweiz: Prof. Dr. Lorenz Hurni. Institut für

Kartografie, ETH Hōnggerberg, 8093 Zürich; hurni@karto.baug.ethz.

ch. Fon: $004116333033 ;$ Fax: 00411631153

Mitarbeit für Österreich: Univ.-Prof. Dr. Wolfgang Kainz.

Institut für Geographie und Regionalforschung, Universität Wien

Universitātsstraße 7/5, 1010 Wien; wolfgang.kainz@univie.ac.at

Fon: 0043 14277-8640; Fax: 0043 14277-9531

Ehrenschriftleiter

Prof, i. R. Dr. Jürgen Dodt, Witten

Dr. rer, nat, h. c. Rolf Harbeck, Bonn

Editorial Board

Prof. Dr. H. Asche, Potsdam; Prof. Dr. Manfred Buchroithner,

Dresden; Prof. Dr. Dirk Burghardt, Dresden; Prof. i. R. Dr. Jürgen

Dodt, Witten; Prof. Dr. D. Dransch, Potsdam; Prof. Dr. M. Ehlers,

Osnabrück, Berlin; Prof. Dr. S. I. Fabrikant, Zürich, Schweiz;

Ass.-Prof. Dr. S. Fuhrmann, Fairfax, USA; Prof. Dr. G. Gartner, Wien. Österreich; Prof. Dr. D. Grünreich, Ronnenberg; Dr. Rolf Harbeck,

Bonn; Prof. Dr. A. Hüttermann, Ludwigsburg; Prof. Dr. L Hurni,

Zürich; Prof. Dr. W. Kainz, Wien; Prof. Dr. P. Kammerer, München;

Prof. Dr. W. G. Koch, Dresden; Prof. Dr. Menno-Jan Kraak, Twente,

Prof. Dr. W. G. Koch, Dresden; Prof. Dr. Menno-Jan Kraak, Twen
Netherlands; Prof. Dr. Jukka Krisp, Augsburg; Prof. Dr.-Ing.

L. Meng, München; Prof. Dr. J. Schiewe, Hamburg; PD Dr.-Ing.

. Schoppmeyer, Bonn; Prof. Dr. J. Schweikart, Berlin; Prof.

Dr.-Ing. M. Sester, Hannover; Prof. Dr. J. Siemer, Regina, Kanada;

Prof. Dr. R. Zölitz, Greifswald.

Manuskripte richten Sie bitte an den zuständigen Schriftleiter.

Hinweise zur Gestaltung des Manuskripts finden Sie unter

www.kartographische-nachrichten.de > Autorenhinweise.

Reviewverfahren

Wissenschaftliche Beiträge werden im Rahmen eines Peer-ReviewVerfahrens begutachtet. Trotz sorgfältiger Redigierung übernehmen

Herausgeber, Redaktion und Verlag keine Haftung für die inhalttiche

Richtigkeit der Veröffentlichungen.

Die KN Kartographische Nachrichten ist in der internationalen Zitationsdatenbank Scopus gelistet.

Die DGfK ist Mitglied der

Internationalen Kartographischen

Vereinigung IKV/ICA

Verlag: Kirschbaum Verlag $\mathrm{GmbH}$, Siegfriedstraße 28 ,

D-53179 Bonn / Postfach 210209, D-53157 Bonn,

Fon: (0228) 95453-0, Fax: (0228) 95453-27:

Internet: http://www.kirschbaum.de, E-Mail: info@kirschbaum.de

Anzeigenleiter: Volker Rutkowski; zurzeit ist Anzeigenpreisliste

Nr. 51 vom 1.10.2015 gültig

Bezugspreise und ISSN: Inland/Ausland Jahresabonnement

inkl. E-Paper und elektronischem Archiv 74, - Euro zzgl.

Versand 9,80,- Euro (jeweils einschl. MwSt.

Einzelheft 18,- Euro zzgl. Versand

Die Zeitschrift erscheint alle zwei Monate. ISSN 0022-9164.

Kündigungsfrist 6 Wochen zum Auslaufen des Abonnement-

Zeitraumes.

Satz: EMS Eckert Medienservice, 53359 Rheinbach

Druck: johnen-druck GmbH \& Co. KG, Industriegebiet Bornwiese,

54470 Bernkastel-Kues

\section{GEOINFO in Potsdam vom 14. bis 16.6.2016}

\section{Liebe Leserin, lieber Leser,}

2016 findet das wichtigste Jahrestreffen der kartographischen Forschung und Praxis in Mitteleuropa, in Potsdam, statt. Potsdam ist bekannt durch zahlreiche wissenschaftliche Einrichtungen mit hervorragendem, weltweitem Ruf, darunter das Potsdam-Institut für Klimafolgenforschung PIK, das Geoforschungszentrum GFZ und natürlich die Universität. Alle diese Institutionen könnten ohne Geoinformationen und Karten nicht arbeiten. Mit den hier tätigen Kartographinnen und Kartographen befinden wir uns mit unserer Jahrestagung in Potsdam

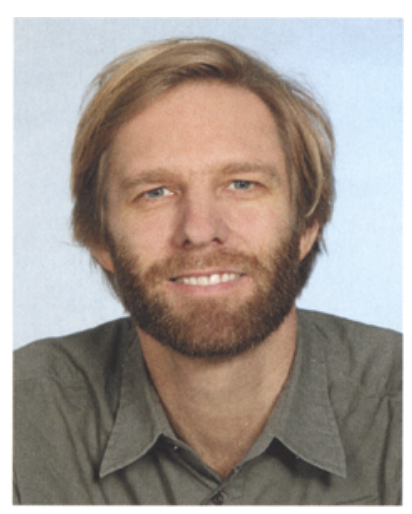

Mark Vetter also in bester professioneller Gesellschaft!

Bei allen Ereignissen dieser Zusammenkunft von Kartographinnen und Kartographen steht sicher die persönliche, fachliche, wissenschaftliche Begegnung im Mittelpunkt - ganz gleich, wie wir die Veranstaltung nennen. Gleichwohl haben wir im Vorstand der DGfK seit Längerem, besonders intensiv aber am 22. April 2016 in Leipzig, über eine Umbenennung unseres Jahresereignisses beraten. Der Präsident, Manfred Weisensee, hat sich diesbezüglich schon in einer Kolumne in KN2/2016 geäußert, der Sekretär, Steffen Hild, hat den Vorschlag zur Satzungsänderung bereits kommuniziert. Letztlich entscheidet aber die Mitgliederversammlung der DGfK. Daher dürfen wir alle sicher sehr gespannt sein, wie die Diskussionen hierzu in Potsdam im Juni 2016 laufen werden.

Die Schriftleitung der $\mathrm{KN}$ hat Ihnen diesmal ein besonders vielfältiges Heft zusammengestellt. Alle Beiträge stehen selbstverständlich im Zusammenhang mit der geoinfo und dem 64. Deutschen Kartographentag in Potsdam. Beate Tomio (ehemals Weninger), Jochen Schiewe und Julius Tröger stellen eine interessante Methode zur Flächeninterpolation für Datenjournalistinnen und -journalisten vor. Andreas Christoph, Wolfgang Crom, Michael Ammon und Holger Przibytzin zeigen in ihrem Beitrag, dass sich mit den heutigen Digitalisierungstechniken fantastische Möglichkeiten für die künftige Führung und Präsentation historischer Karten ergeben. Anja Reinermann-Matatko hat in ihrem Beitrag über Kommunalfinanzen in Karten den Zusammenhang zwischen Kommunalplanung und empirischer Kartographie dargestellt. Schließlich bringen uns Malte Schulze und Frank Thiemann näher, was wir in Zukunft im Rahmen der automatisierten geometrischen Anpassung von Katasterdatensätzen erwarten dürfen.

Ich wünsche Ihnen viel Freude beim Lesen der Kartographischen Nachrichten, und ich freue mich auf ein Treffen mit Ihnen in Potsdam,

herzlichst,

Ihr



